\title{
Phagocytosis by pig alveolar macrophages of Actinobacillus pleuropneumoniae serotype 2 mutant strains defective in haemolysin II (ApxII) and pleurotoxin (ApxIII)
}

\author{
Janice M. Cullen and Andrew N. Rycroft $\dagger$ \\ Author for correspondence: Janice M. Cullen. Tel: +44 413398855 . Fax: +44 413305602.
}

Department of Veterinary Pathology, University of Glasgow, Glasgow G61 1QH, UK
The ability of pig alveolar macrophages to phagocytose Actinobacillus pleuropneumoniae HK 361, which produces both haemolysin II (ApxII) and pleurotoxin (ApxIII), has been studied. Macrophages incubated with HK 361 in the presence of normal pig serum were rapidly killed. Incubation of the macrophages with a haemolysin-deficient mutant (HK 361 e), which possesses only cytotoxic activity (ApxIII), also caused gross damage to the macrophages. A mutant (HK 361 h) which produces neither Apxll nor ApxIII in its culture supernatant allowed longer survival of the macrophages than did either the parent strain or mutant e when incubated with normal pig serum. Prolonged incubation with mutant $h$ resulted in an increase in the number of damaged macrophages, but not to the same extent as with either HK 361 or mutant e. The number of mutant $h$ cells phagocytosed in the presence of normal pig serum was low. The addition of either hyperimmune rabbit serum, raised against whole formalin-treated HK 361 cells, or convalescent pig serum from a pig recovering from a serotype 3 infection, which contained antibody against both ApxII and ApxIII, did not increase the survival of macrophages incubated with either HK 361 or mutant e. However, incubation of mutant $h$ with convalescent pig serum did result in damage-free macrophages. This serum, which possessed neutralizing capabilities against the toxic activities of ApxII and ApxIII, enhanced the number of mutant $h$ cells phagocytosed compared to the numbers phagocytosed in normal pig serum. Killed bacteria were rapidly phagocytosed and did not damage macrophages. The number of phagocytosed killed bacteria appeared to be similar to that seen with live mutant $h$ cells when incubated in the presence of convalescent pig serum. The presence of a toxic activity associated with the haemolysin- and cytotoxin-negative mutant may represent an additional cell-associated toxin which is not present in its culture supernatant. It appears therefore, that in the absence of extracellular ApxII and ApxIII and in the presence of convalescent pig serum $A$. pleuropneumoniae is readily phagocytosed.

Keywords: Actinobacillus pleuropneumoniae mutants, phagocytosis, pig alveolar macrophages, toxins

\section{INTRODUCTION}

Actinobacillus pleuropneumoniae is the causative agent of porcine pleuropneumonia, a highly contagious and poten-

\footnotetext{
† Present address: Department of Pathology and Infectious Diseases, The Royal Veterinary College, University of London, London NW1 OTU, UK. Abbreviation: PMN, polymorphonuclear leucocytes.
}

tially fatal pig disease (Nicolet, 1992). Infection occurs via the respiratory route by inhalation of aerosol droplets containing the bacterium (Sebunya \& Saunders, 1983; Nicolet, 1992). Clinical signs of porcine pleuropneumonia range from chronic to peracute and are characterized by haemorrhagic, fibrinous lesions of the respiratory tract (Nicolet, 1992). Toxins have been postulated to be involved in the rapid onset of clinical signs, following the discovery that bacteria-free culture supernatant could 
produce lesions similar to those caused by natural infection (Rosendal et al., 1980). These extracellular toxins have been reported to be toxic for phagocytic cells (Bendixen et al., 1981; Udeze \& Kadis, 1988; Rosendal et al., 1988; Van Leengoed et al., 1989) and to possess haemolytic activity (Nakai et al., 1983; Kume \& Nakai, 1986; Rosendal et al., 1988; Frey \& Nicolet, 1990; Kamp et al., 1991). Three toxin proteins have been identified in the culture supernatants of the 12 serotypes of $A$. pleuropneumoniae. They have previously been named differently by the various research groups and include HlyI (Frey \& Nicolet, 1990) and ClyI (Kamp et al., 1991), HlyII (Frey et al., 1992) and ClyII (Kamp et al., 1991), and lastly, Ptx (Rycroft et al., 1991) and ClyIII (Kamp et al., 1991). Recently the nomenclature of the three toxins has been standardized to ApxI, ApxII and ApxIII, respectively (Frey et al., 1993).

Alveolar macrophages form a major part of the defence system of the lung against bacterial infection. The damage caused to these cells by $A$. pleuropneumoniae toxins may reduce the efficiency of this system. This is of obvious advantage to invading bacteria, aiding their colonization and ability to cause disease. The mechanism by which these toxins damage phagocytic cells is unknown; however, it has been shown that ApxI of the serotype 1 strain 4074 forms pores in phospholipid membranes (Lalonde $e t$ al., 1989). Phagocytosis of $A$. pleuropneumoniae has been documented using porcine polymorphonuclear leucocytes (PMN) and alveolar macrophages (Inzana et al., 1988; Udeze \& Kadis, 1988; Thwaits \& Kadis, 1991). There have also been reports of damage to these cell types by the same A. pleuropneumoniae strains (Pijoan, 1986). These contradictory reports of damage to the phagocytic cells and also of their ability to phagocytose adequately left the situation unclear as to the actual extent of damage caused by $A$. pleuropneumoniae to phagocytic cells.

Phagocytosis of $A$. pleuropneumoniae has been studied only in strains that produce both ApxI and II (Inzana et al., 1988; Udeze \& Kadis, 1988; Thwaits \& Kadis, 1991). We have previously found that a serotype 2 strain, HK 361 , produces a potent cytotoxin associated with a $120 \mathrm{kDa}$ protein (ApxIII, formerly Ptx) which is active against alveolar macrophages (Rycroft et al., 1991). However, it was not known whether macrophages are able to phagocytose prior to being killed. The aim of this study was therefore to evaluate whether phagocytosis by alveolar macrophages occurred in the presence of both ApxII and ApxIII, and, secondly, whether phagocytosis was occurring in the absence of ApxII and ApxIII using mutants deficient in either ApxII alone or both ApxII and ApxIII.

\section{METHODS}

Bacterial strains and culture conditions. $A$. pleuropneumoniae strain HK 361 was obtained from the National Collection of Type Cultures (Colindale, UK). HK 361 mutants e and h have been described previously (Rycroft et al., 1991). Mutant e possesses cytotoxin activity only, associated with a $120 \mathrm{kDa}$ protein (ApxIII, formally pleurotoxin), and mutant $h$ is deficient in both ApxII (formally haemolysin type II, $109 \mathrm{kDa}$ protein) and ApxIII. No other phenotypic differences were found. Growth rates, colony morphology, outer-membrane-protein profiles and lipopolysaccharide of both mutants were found to be identical to the parent strain. Eschericbia coli RY22 was also used (Rycroft et al., 1983). A. pleuropneumoniae strains were stored in $10 \%(\mathrm{w} / \mathrm{v})$ skimmed milk at $-70{ }^{\circ} \mathrm{C}$. Routine culture was made on heated blood agar (prepared with $7 \%, w / v$, horse blood), and liquid cultures were grown in Tryptone Soya Broth (TSB; Oxoid) supplemented with $2 \mu \mathrm{g}$ NAD $\mathrm{ml}^{-1}$ (Sigma) (TSB/NAD). E. coli strain RY22 was stored on Dorset Egg slopes. Routine culture was made on nutrient agar and liquid cultures were also grown in TSB. A. pleuropneumoniae was grown overnight on solid medium followed by overnight culture with aeration (100 r.p.m.) in TSB/NAD. Subcultures were then made ( 1 in 25 ) into fresh TSB/NAD and grown for a further $2 \mathrm{~h}$ until the exponential growth phase was reached. Growth of bacterial cultures was determined by measuring the $\mathrm{OD}_{650}$ using a Beckman DU 64 spectrophotometer; an $\mathrm{OD}_{650}$ of 0.1 corresponded to $10^{8}$ A. pleuropneumoniae organisms $\mathrm{ml}^{-1}$.

Inactivation of bacteria. Bacteria were grown as previously described, and treated either for $2 \mathrm{~min}$ at $100{ }^{\circ} \mathrm{C}$ or with $0.5 \%$ $(\mathrm{v} / \mathrm{v})$ formalin overnight to inactivate the bacteria.

Sera. Blood samples were obtained from normal pigs by aseptic venepuncture of the cranial vena cava. The blood was allowed to clot at $37^{\circ} \mathrm{C}$ for $30 \mathrm{~min}$ followed by $30 \mathrm{~min}$ on ice. Serum was separated following centrifugation at $2000 \mathrm{~g}$ at $4{ }^{\circ} \mathrm{C}$, stored at $-70^{\circ} \mathrm{C}$, and working volumes were held at $4{ }^{\circ} \mathrm{C}$. Normal pig serum contained no detectable agglutinating or reacting antibodies against $A$. pleuropneumoniae, as measured by the standard tube agglutination of whole bacteria (Cruikshank, 1962) and whole bacteria ELISA (Loftager et al., 1993). Hyperimmune rabbit serum was raised in New Zealand White rabbits by repeated intravenous inoculation, using standard methods (Harlow \& Lane, 1988), with formalin-killed cells of HK 361 which had been treated with $0.5 \%(\mathrm{v} / \mathrm{v})$ formalin overnight and washed three times in phosphate-buffered saline. Convalescent pig serum was obtained from a pig with a natural chronic infection caused by a serotype 3 strain, 6664 (Rycroft \& Cullen, 1990). Heat-inactivated serum was treated at $56^{\circ} \mathrm{C}$ for $30 \mathrm{~min}$.

Alveolar macrophages. Lungs were obtained from high-healthstatus pigs which were killed with barbiturates and used immediately. Lung lavage was performed using Hanks' Balanced Salt Solution (HBSS; Gibco), which was placed in the trachea by means of a filter funnel. The lungs were inflated to maximum capacity with HBSS and gently massaged. The HBSS was then emptied into a sterile container held on ice and the procedure repeated using fresh HBSS. The suspension collected in this way was then centrifuged at $110 \mathrm{~g}$ for $4 \mathrm{~min}$ and the pellet washed twice with ice-cold HBSS containing $0.5 \%$ gelatin (gelHBSS) at $4{ }^{\circ} \mathrm{C}$ (Leijh et al., 1986; Rycroft et al., 1991). Gelatin was added to protect the bacteria in the phagocytosis assay, since HBSS alone is bactericidal (Roberts, 1967). Remaining red cells were lysed by the addition of $0.87 \%(w / v) \mathrm{NH}_{4} \mathrm{Cl}$ for 10 min. Following centrifugation, the cell pellet was resuspended in gel-HBSS, and the viability of the cell suspension determined by the trypan-blue exclusion test. The cell suspension was adjusted to $1 \times 10^{7}$ viable cells $\mathrm{ml}^{-1}$, and Giemsastained cell preparations were examined for cell type by light microscopy.

Phagocytic assay. The method used was based upon that of Leijh et al. (1986). Bacteria, grown or treated as previously described, were washed once in gel-HBSS and resuspended in the same buffer to $5 \times 10^{7}$ cells $\mathrm{ml}^{-1}$. A portion $(0.5 \mathrm{ml})$ of bacterial suspension was incubated with $0.5 \mathrm{ml}$ macrophages 

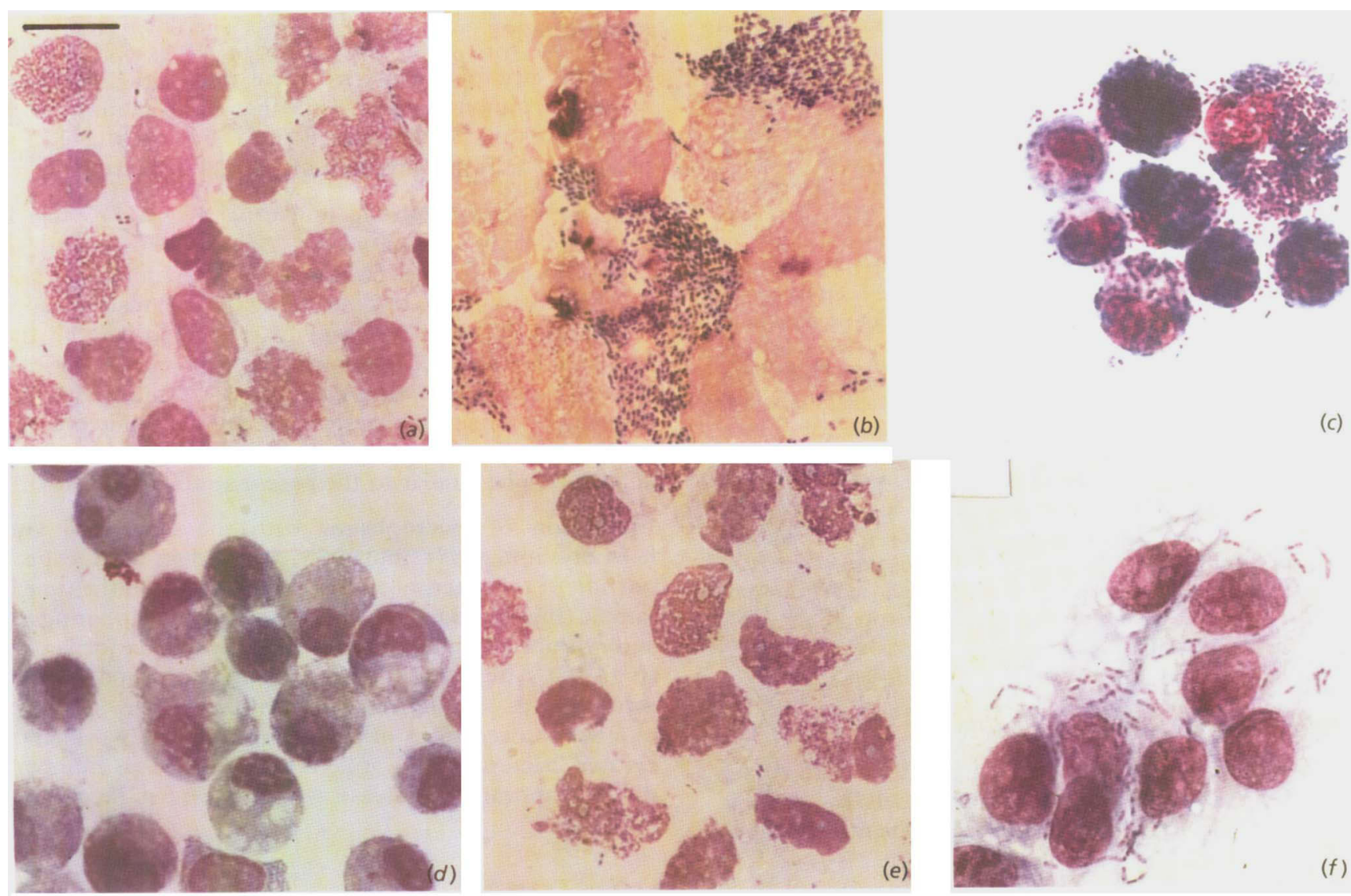

(c)
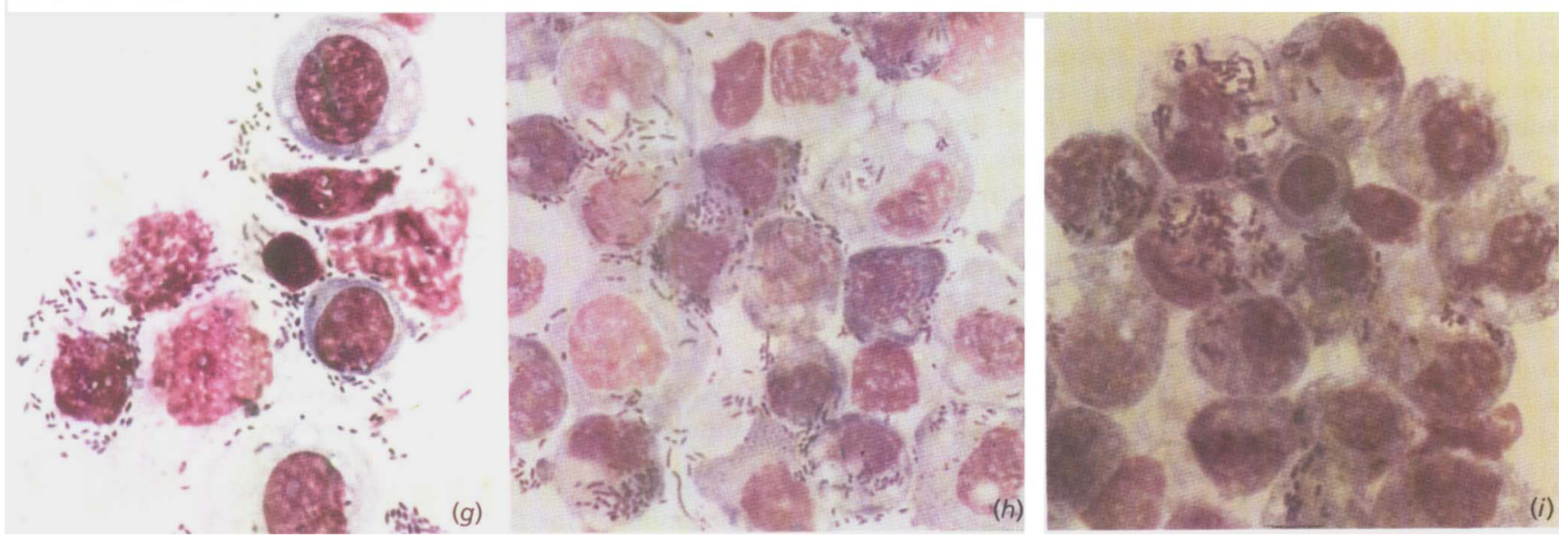

Fig. 1. Cytospin preparations of porcine alveolar macrophages incubated with $A$. pleuropneumoniae were assessed at various time intervals for both phagocytosis of bacteria and survival of macrophages (bar, $50 \mu \mathrm{m})$. The magnification is the same for each photograph. (a) $\mathrm{HK} 361$ (ApxII+ ApxIII+) $t=0$ sample incubated with normal pig serum; (b) $\mathrm{HK} 361$ $\left(\right.$ ApxII ${ }^{+}$ApxIII ${ }^{+}$) $1 \mathrm{~h}$ sample incubated with normal pig serum; (c) $E$. coli RY22 control incubated with heated normal pig serum at $2 \mathrm{~h}$; (d) macrophages incubated in the absence of bacteria with normal pig serum at $2 \mathrm{~h}$; (e) HK 361 mutant e (ApxII ${ }^{-}$ApxIII ${ }^{+}$) incubated with normal pig serum at $t=0$; (f) HK 361 mutant $h$ (ApxII- ApxIII-) incubated with normal pig serum at $t=0 ;(\mathrm{g}) \mathrm{HK} 361$ mutant $\mathrm{h}$ (ApxII- ApxIII-) incubated with normal pig serum at $2 \mathrm{~h}$; (h) HK 361 mutant $\mathrm{h}$ (ApxIIApxIII-) incubated with immune pig serum at $2 \mathrm{~h}$; (i) killed $\mathrm{HK} 361$ mutant $h$ (ApxII- ApxIII-) incubated with normal pig serum at $2 \mathrm{~h}$. Each sample was taken in either duplicate or triplicate and each experiment was carried out between three and five times. 
$\left(1 \times 10^{7}\right.$ cells $\left.\mathrm{ml}^{-1}\right)$ and $0 \cdot 1 \mathrm{ml}$ serum in non-adherent tubes (Leijh et al., 1986) at $37^{\circ} \mathrm{C}$ with slow rotation (4 r.p.m.). Samples were taken at 0,1 and $2 \mathrm{~h}$; viable bacterial counts and preparations for cytospins and electron microscopy were carried out by the methods described below. Bacteriological determination of undigested unattached viable bacteria was carried out as follows. Samples $(100 \mu \mathrm{l})$ were transferred to $300 \mu \mathrm{l}$ icecold gel-HBSS to stop phagocytosis and centrifuged at $110 \mathrm{~g}$ for 4 min at $4{ }^{\circ} \mathrm{C}$. Supernatants were carefully aspirated and stored. Pellets were washed twice more using $300 \mu \mathrm{l}$ gel-HBSS each time, giving a total of $1 \mathrm{ml}$ of washings. A portion $(10 \mu \mathrm{I})$ of this supernatant was diluted $10^{-2}$ and $10^{-4}$ in gel-HBSS. Samples $(100 \mu \mathrm{l})$ of each dilution were assessed for viable bacteria by the pour-plate method. The pellets were then used to prepare both cytospin smears and sections for examination by electron microscopy. Pellets were resuspended in $400 \mu \mathrm{l}$ gel-HBSS. For cytospins, a portion $(200 \mu \mathrm{l})\left(4.5 \times 10^{5}\right.$ cells $)$ of each sample was centrifuged for $5 \mathrm{~min}$ at $55 \mathrm{~g}$ in a cytocentrifuge (Shandon). Slides were air dried, fixed with methanol and stained with Giemsa solution. The remaining $200 \mu$ l samples were used for electron microscopy. Samples were fixed with $1.3 \%$ paraformaldehyde and $1.6 \%$ glutaraldehyde in $0.1 \mathrm{M}$ sodium cacodylate. They were then postfixed with osmium tetroxide, sectioned, stained with $20 \%$ uranyl acetate and lead citrate and examined with a transmission electron microscope (Zeiss 109).

\section{RESULTS}

\section{Phagocytosis of A. pleuropneumoniae}

A. pleuropneumoniae $\mathrm{HK} 361$, which produces both ApxII and ApxIII, was assessed for phagocytosis by alveolar macrophages following incubation with the macrophages in the presence of normal pig serum.

Lung lavage samples were examined by light microscopy and found to be almost entirely composed of alveolar macrophages $(>99 \%)$. Following exposure of the macrophages to $A$. pleuropneumoniae HK 361 in the presence of normal pig serum, the majority of the cells observed in the cytospin preparations had already undergone a degree of degeneration in the time 0 sample, which was collected immediately after the addition of the bacteria to the macrophage and serum mixture (Fig. 1a). In the presence or absence of normal pig serum, similar macrophage degeneration occurred. In the presence of serum, bacteria were seen to adhere to the macrophage surface, and occasionally bacteria were present within phagolysosomes. No bacteria were seen to be either phagocytosed or adhering to the macrophages in the absence of serum. After further incubation for 1 and $2 \mathrm{~h}$, all macrophages displayed extensive damage in both the presence and absence of serum (Fig. 1b). Bacteria appeared to be located both around and within the dead macrophages, again only in the presence of serum. The numbers of bacteria observed in the cytospin preparations increased greatly with continued incubation (Fig. 1b).

Control macrophage samples, incubated either with $E$. coli RY22 (Fig. 1c) or in the absence of bacteria (Fig. 1d), showed no signs of damage at any stage of incubation. Large numbers of $E$. coli were seen to be phagocytosed after 1 and $2 \mathrm{~h}$ incubation in the presence of serum (Fig. 1c).
Effect of A. pleuropneumoniae haemolysin (ApxII)and cytotoxin (ApxIII)-negative mutants on alveolar macrophages

Alveolar macrophages were incubated with $A$. pleuropneumoniae mutants negative in either ApxII alone or both ApxII and ApxIII. Mutants $e$ and $h$ were evaluated for phagocytosis in the presence of normal pig serum.

The haemolysin-deficient mutant, HK 361 e, which possessed only the cytotoxin (ApxIII), was found to behave in an identical fashion to the parent strain, HK 361. Incubation of this strain with the macrophages resulted in the rapid death of macrophages in both the presence and absence of serum (Fig. 1e). The increase in bacterial numbers over time, seen associated with the degenerating macrophages in the presence but not the absence of normal pig serum in the cytospin preparations, appeared to be similar to the parent strain, HK 361.

Incubation of macrophages with the cytotoxin- and haemolysin-negative mutant $h$, resulted in a large proportion of the macrophages remaining alive in the time 0 sample (Fig. 1f). Bacteria were observed both adhering to, and within, the macrophages in the presence of serum (Fig. 1f). After 1 and $2 \mathrm{~h}$ incubation, a proportion of macrophages still appeared healthy; however, a similar number showed signs of degeneration (Fig. 1g). Phagocytosed bacteria could still be seen, although numbers did not appear to increase with prolonged incubation (Fig. 1g).

\section{Effects of immune sera on macrophage survival and ability to phagocytose}

Phagocytosis assays were also carried out in the presence of immune sera which contained antibodies against ApxII and ApxIII. This was to assess, firstly, any increase in survival of macrophages incubated with $A$. pleuropneumoniae due to the presence of neutralizing antibodies against the ApxII and ApxIII and, secondly, any increase in the number of phagocytosed $A$. pleuropneumoniae when incubated with immune sera. HK 361 and mutants e and $h$ were assessed for phagocytosis by alveolar macrophages incubated with both convalescent pig serum and hyperimmune rabbit serum.

No difference in the degree of damage caused to the macrophages incubated with $\mathrm{HK} 361$ and mutant e was seen when the assays were incubated with either of the immune sera. Macrophages incubated with HK 361 and mutant e continued to sustain extensive damage. Preopsonization of $\mathrm{HK} 361$ bacteria for $30 \mathrm{~min}$ at $37^{\circ} \mathrm{C}$ prior to incubation with the macrophages did not reduce the level of damage to macrophages.

Incubation of mutant $\mathrm{h}$ with immune sera resulted in the macrophages initially appearing healthy. Continued incubation in the presence of hyperimmune rabbit serum resulted in the killing of a proportion of macrophages comparable to that seen with normal pig serum. The presence of convalescent pig serum, however, did appear to reduce damage to the macrophages, resulting in most 

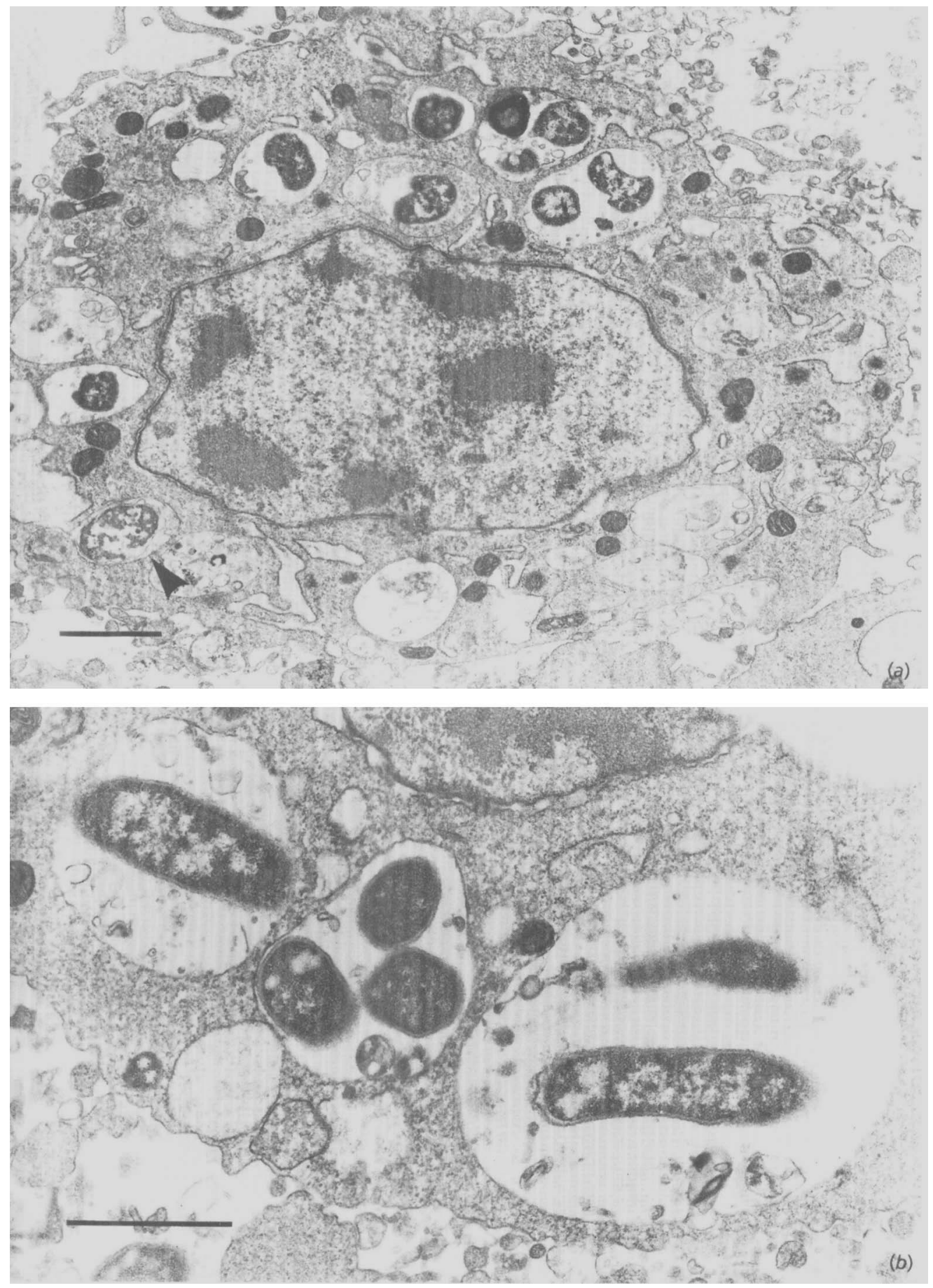

Fig. 2. Electron micrographs of $A$. pleuropneumoniae mutant $h$ (ApxII- $A p x \mid I^{-}$), opsonized with immune pig serum, within phagolysosomes of pig alveolar macrophages. (a) Bacteria can be seen within several phagolysosomes in a single alveolar macrophage. The arrow points to a phagolysosome within which a bacterium appears to have undergone a degree of degeneration. (b) The double membranes of the bacteria are clearly visible. Bars, $1 \mu \mathrm{m}$. 
of the cells remaining healthy following continued incubation (Fig. 1h). Bacteria could be clearly seen adhering to macrophages and within phagolysosomes (Fig. 1h). The numbers of phagocytosed bacteria also appeared to increase with prolonged incubation. Phagocytosis of mutant $\mathrm{h}$ was confirmed following electron microscopy. Bacteria could be seen within phagolysosomes (Fig. 2a). A double membrane could be clearly seen surrounding several of the bacteria (Fig. 2b).

In control samples where mutant $h$ was incubated with macrophages in the absence of serum, a slight reduction in the level of toxicity was observed after $1 \mathrm{~h}$ incubation compared to all other serum conditions, excluding the convalescent serum. This difference could only be seen in the $1 \mathrm{~h}$ sample. After $2 \mathrm{~h}$ incubation, the macrophages were at similar levels of degeneration in all the other serum conditions except the convalescent serum. Controls of macrophages incubated in the presence and absence of E. coli bacteria showed no signs of damage as previously described (Fig. 1c, d).

\section{Phagocytosis of inactivated A. pleuropneumoniae}

Both toxins produced by HK 361 are heat labile (Rycroft et al., 1991). Other researchers have documented, from a different serotype 2 strain, a heat-stable toxin(s) that possessed both haemolytic and cytotoxic properties and was polysaccharide in nature (Kume \& Nakai, 1986). To assess whether the damage seen to macrophages incubated with mutant $h$ in the presence of normal pig serum was related to such a factor, bacteria inactivated by heat or formaldehyde were assessed for phagocytosis.

HK 361 mutant h bacteria, killed either by heat or by formaldehyde, appeared to be readily phagocytosed by the macrophages in the presence of each serum used. In the time 0 samples, bacteria could already be clearly seen inside the macrophages. After incubation for $2 \mathrm{~h}$, the number of phagocytosed bacteria had increased substantially compared to the first sample (Fig. 1i). No damage to the macrophages was apparent in any of the later samples. No difference in the degree of phagocytosis of $A$. pleuropneumoniae was detected when incubated in the presence of either normal or immune pig serum. All macrophage controls were as previously described.

\section{Determination of extracellular viable bacterial counts}

Reproducible counts of extracellular bacteria could not be determined for $A$. pleuropneumoniae. The ability of A. pleuropneumoniae to form chains, and the adherence of large numbers of bacteria to the degenerate macrophages, even after three slow-speed washes to remove extracellular bacteria, may have contributed to the observed irregularities. Reproducible extracellular bacterial counts were obtained for the E. coli controls. Bacterial counts decreased with increased incubation time in the presence of both macrophages and serum. Increases in extracellular bacterial counts over time were observed for the serumfree control, and also in the macrophage-free control both in the presence and absence of serum. The increase in bacterial counts was very similar for the above three controls.

\section{DISCUSSION}

The results obtained to date regarding phagocytosis of A. pleuropneumoniae strains have been documented with strains that produce both ApxI and ApxII (Inzana et al., 1988; Udeze \& Kadis, 1988, 1992; Thwaits \& Kadis, 1991). Strains of serotypes 2, 3, 4, 6 and 8 also produce a $120 \mathrm{kDa}$ protein (Cullen \& Rycroft, 1992; Macdonald \& Rycroft, 1992) which is associated with cytotoxicity (Rycroft et al., 1991). The aim of this work was to assess whether a serotype 2 strain of $A$. pleuropneumoniae is phagocytosed by alveolar macrophages in the presence or absence of toxic activities ApxII and ApxIII.

Phagocytosis of HK 361 or mutant e could not be assessed due to the rapid toxic effect of these organisms on the macrophages. The addition of immune serum with or without preopsonization for $30 \mathrm{~min}$ at $37^{\circ} \mathrm{C}$ did not remove this toxic effect. The difference observed in the neutralizing ability of the immune serum compared to that previously seen (Rycroft et al., 1991) may be related to the continual production of toxic activity by the growing bacteria during the phagocytic assay. A fixed amount of toxin was present in the culture supernatant used in the previous study. Continued production may allow the toxin to overcome the neutralizing ability of serum antibody.

The absence of ApxII in mutant e appeared not to reduce the level of macrophage damage, suggesting it was not the only cause of the observed bacterial toxicity. This is in agreement with work of others, who have found the ApxII to be weakly haemolytic and the least cytotoxic of the three known cytotoxins (ApxI, II and III) (Frey \& Nicolet, 1990; Rycroft et al., 1991; Smits et al., 1991; Kamp et al., 1991). The limited macrophage damage observed after 1 and $2 \mathrm{~h}$ incubation with mutant $\mathrm{h}$ may be due to the production by this mutant of a lower level of toxic activity not detectable in our assays, as culture supernatant from mutant $h$ has previously been found to be non-toxic for alveolar macrophages, and neither ApxII nor ApxIII was detected in the culture supernatant by immunoblotting (Rycroft et al., 1991). It may also be speculated that the reduced toxic activity observed in A. pleuropneumoniae mutant $\mathrm{h}$ represents some other cellassociated toxic component.

Only in the presence of convalescent pig serum was the toxicity of mutant $h$ for macrophages neutralized. The lack of neutralization of toxic activity by the hyperimmune rabbit serum, which contained cross-reacting but not neutralizing antibodies against both ApxII and ApxIII (personal observations), may also imply the involvement of toxic activities in the macrophage damage by mutant $h$. The increase in phagocytosed mutant $h$ following prolonged incubation in the presence of the immune pig serum suggests the toxin-neutralizing abilities of the convalescent pig serum are required to enhance the phagocytosis of this organism. Whether this is a result of 
the neutralizing abilities of the immune serum alone, or when in combination with the presence of specific antibodies which have been reported to enhance opsonization of $A$. pleuropneumoniae, is not known (Thwaits \& Kadis, 1991). The rapid phagocytosis of the killed bacteria, and the lack of damage to the alveolar macrophages incubated in normal pig serum, suggest that $A$. pleuropneumoniae is phagocytosed in the absence of any heat- or formaldehyde-sensitive extracellular toxins. The phagocytosis of killed mutant $h$ also suggests that the toxic activity of this mutant is heat labile and therefore not LPS associated, nor is it the same as the heat-stable toxin described by Kume \& Nakai (1986).

Attempts were made to assess whether phagocytosed mutant h cells were killed by macrophages. However, the use of the detergent Triton $\mathrm{X}$ at a concentration which lysed the macrophages $(0 \cdot 1 \%)$ was found to be toxic for the bacteria. Rapid freeze/thawing techniques in the presence of bovine serum albumin $(1 \%)$ also killed the bacteria. However, it is of interest that, in Fig. 2, as indicated by an arrow, a mutant h bacterium within the phagolysosome appears to have undergone a degree of degeneration. Whether this demonstrates killing of mutant $\mathrm{h}$ within alveolar macrophages, or is merely an artifact of the electron microscopy, remains unclear.

A. pleuropneumoniat HK 361 and its mutants were found to remain attached to both the viable and the degenerate macrophages after washing to remove extracellular bacteria. This occurred only in the presence of serum, irrespective of whether it had been heated or contained specific antibodies. Both Thwaits \& Kadis (1991) and Inzana et al. (1988) recovered high percentages of total bacteria in the supernatant following three slow-speed washes to remove extracellular bacteria in a procedure identical to that used here. Both these researchers used PMN and perhaps the differences can be attributed to this factor. This problem of adherence was not detected using the $E$. coli control. The lack of reproducible viable bacterial counts would also probably be due to the adherence of bacteria to the macrophages following washing.

Phagocy tosis of live A. pleuropneumoniae by PMN has been described for strains 4074 (serotype 1) and K17 (serotype 5a) that produced ApxI and II (Inzana et al., 1988; Thwaits \& Kadis, 1991). ApxI is reported to possess strong cytocidal activity, and ApxII a weaker activity (Kamp et al., 1991). However, toxicity to alveolat macrophages has also been caused by the same strains (Pijoan, 1986). These differences may have been related to these two strains being toxic for porcine alveolar macrophages but not for PMN. A third study used porcine alveolar macrophages to study phagocytosis of serotype 1 strain 4074 (Ude\% \& Kadis, 1988). They did report toxicity of the puritied ApxI for alveolar macrophages and PMN alike; however there was no discussion of whether toxicity was observed in the phagocytosis assays with whole bacteria. Recent studies with the same strain, 4074, have since found that this strain was in fact toxic for PMN (Udeze \& Kadis, 1992). These researchers found that substantial phagocytosis could only be achieved when the assay was carried out in the presence of serum containing antibodies to ApxI and II. It would therefore appear that this strain (4074), producing ApxI and II, is toxic for both PMN and macrophages alike; however the presence of immune serum neutralizes the toxic effect of the bacteria and phagocytosis can therefore be assessed. This would explain why there have been contradictory reports of damage to phagocytic cells by both culture supernatant and live bacteria, which were assessed in the absence of immune serum, and phagocytosis of the same strains, which were assessed in the presence of immune serum. We have found that even in the presence of immune serum the toxic action of viable strains producing ApxIII for pig alveolar macrophages cannot be neutralized.

The delayed death of the macrophage in the serum-free control may suggest that attachment of the organism to the macrophages enhances the action of the toxic components. It has been suggested that the toxin operates by a diffusion mechanism (Udeze \& Kadis, 1988).

In summary, the results of the investigation suggest that phagocytosis of $A$. pleuropneumoniae serotype 2 strain by alveolar macrophages can only occur in the absence of both ApxII and ApxIII and in the presence of immune serum. When ApxIII is present, either alone (mutant e) or in combination with ApxII (HK 361), phagocytosis cannot be assessed. This is due to the extremely toxic activity of the ApxIII, which cannot be entirely neutralized by immune serum when live bacteria are used. We therefore suggest that $A$. pleuropneumoniae would be phagocytosed except for the fact that it produces toxins active against phagocytic cells.

\section{ACKNOWLEDGEMENTS}

We acknowledge the photographic assistance of Allan May and Colin Wilson. We are grateful to Ross Blackley and Celia Burke for their technical abilities in performing the electron microscopy, and to J. Macdonald and D. J. Taylor for their helpful advice on the manuscript.

This work was supported by the Agricultural and Food Research Council, Grant AG17/201, and the Wellcome Trust Grant 030536.

\section{REFERENCES}

Bendixen, P. H., Shewen, P. E., Rosendal, S. \& Wilkie, B. N. (1981). Toxicity of Haemophilus pleuropneumoniae for porcine lung macrophages, peripheral blood monocytes, and testicular cells. Infect Immun 33, 673-676.

Cruikshank, R. (editor) (1962). Mackie \& McCartney's Handbook of Bacteriology. A Guide to Laboratory Diagnosis and Control of Infection, 10th edn, pp. 313-332. Edinburgh \& London: E. \& $S$. Livingstone.

Cullen, J. M. \& Rycroft, A. N. (1992). Identification and cross reactivity of the haemolysin and cytotoxin proteins of Actinobacillus pleuropneumoniae and related pathogens by the use of monoclonal antibodies. Proc Int Pig Vet Soc Congr 12, 198.

Frey, J. \& Nicolet, J. (1990). Hemolysin patterns of Actinobacillus pleuropneumoniae. I Clin Microbiol 28, 232-236. 
Frey, J., van den Bosch, H., Segers, R. \& Nicolet, J. (1992). Identification of a second hemolysin (HlyII) in Actinobacillus pleuropneumoniae serotype 1 and expression of the gene in Escherichia coli. Infec Immun 60, 1671-1676.

Frey, J., Bosse, J. T., Chang, Y.-F., Cullen, J. M., Fenwick, B., Gerlach, G. F., Gygi, D., Haesebrouck, F., Inzana, T. J., Jansen, R., Kamp, E. M., Macdonald, J., Maclnnes, J. I., Mittal, K. R., Nicolet, J., Rycroft, A., Segers, R. P. A. M., Smits, M. A., Stenbaek, E., Struck, D. K., van den Bosch, J. F., Willson, P. J. \& Young, R. (1993). Actinobacillus pleuropneumoniae R'TX-toxins: uniform designation of haemolysins, cytolysins, pleurotoxin and their genes. $J$ Gen Microbiol 139, 1723-1728.

Harlow, E. \& Lane, D. (1988). Antibodies: a Laboratory Manual. Cold Spring Harbor, NY: Cold Spring Harbor Laboratory.

Inzana, T. J., Ma, J., Workman, T., Gogolewski, R. P. \& Anderson, P. (1988). Virulence properties and protective efficiency of the capsular polymer of Haemopbilus (Actinobacillus) pleuropneumoniae serotype 5. Infect Immun 56, 1880-1889.

Kamp, E. M., Popma, J. K., Anakotta, J. \& Smits, M. A. (1991). Identification of hemolytic and cytotoxic proteins of Actinobacillus pleuropneumoniae by use of monoclonal antibodies. Infect Immun 59, 3079-3085.

Kume, K. \& Nakai, T. (1986). Species specificity of Haemophilus pleuropneumonia hemolysin demonstrated in the cytocidal and antiphagocytic effects. Jpn J Vet Sci 48, 993-997.

Lalonde, G., McDonald, T. V., Gardner, P. \& O'Hanley, P. D. (1989). Identification of a hemolysin from Actinobacillus pleuropneumoniae and characterisation of its channel properties in planar phospholipid bilayers. J Biol Cbem 264, 13559-13564.

Leijh, P. C. J., Van Furth, R. \& Van Zwet, T. L. (1986). In vitro determination of phagocytes and intracellular killing by polymorphonuclear and mononuclear phagocytes. In Handbook of Experimental Immunology, vol. 2, Cellular Immunology, 4th edn, pp. 46.1-46.21. Edited by D. M. Weir. Oxford: Blackwell Scientific Publications.

Loftager, M. K., Eriksen, L. \& Nielsen, R. (1993). Antibodies against Actinobacillus pleuropneumoniae serotype 2 in mucosal secretions and sera of infected pigs as demonstrated by an enzyme-linked immunosorbent assay. Res Vet Sci 54, 57-62.

Macdonald, J. \& Rycroft, A. N. (1992). Molecular cloning and expression of ptx $A$, the gene encoding the 120 -kilodalton cytotoxin of Actinobacillus pleuropneumoniae serotype 2. Infect Immun 60, 2726-2732.

Nakai, T., Sawata, A. \& Kume, K. (1983). Characterization of the hemolysin produced by Haemophilus pleuropneumoniae. Am J Vet Res 44, $344-347$.

Nicolet, J. (1992). Haemopbilus infections. In Diveases of Swine, pp. 426 433. Edited by A. D. Leman, B. Straw, R. D. Glock, W. L.
Mengeling, R. H. C. Penny \& E. Scholl. Ames: Iowa State University Press.

Pijoan, C. (1986). Effects of Pasteurella multocida and Haemophilus pleuropneumonia toxins on swine alveolar macrophages. Vet Immunol Immunopatbol 13, 141-149.

Roberts, R. B. (1967). The interaction in vitro between group B meningococci and rabbit polymorphonuclear leukocytes. Demonstration of type specific opsonins and bactericidins. J Exp Med 126, 795-799.

Rosendal, S., Mitchell, W. R., Weber, M., Wilson, M. R. \& Zaman, M. R. (1980). Hemophilus pleuropneumonia lung lesions induced by sonicated bacteria and sterile culture supernatant. Proc Int Pig Vet Soc Congr 5, 221.

Rosendal, S., Devenish, J., Maclnnes, J. I., Lumsden, J. H., Watson, S. \& Xun, H. (1988). Evaluation of heat-sensitive, neutrophil-toxic, and hemolytic activity of Actinobacillus (Haemophilus) pleuropneumoniae. Am J Vet Res 49, 1053-1058.

Rycroft, A. N. \& Cullen, J. M. (1990). Complement resistance in Actinobacillus (Haemopbilus) pleuropneumoniae infection in swine. Am J Vet Res 51, 1449-1453.

Rycroft, A. N., Thompson, G. \& Hammond, S. M. (1983). The role of cell surface polysaccharide antigens in the pathogenicity of Escherichia coli. FEMS Microbiol Lett 18, 49-53.

Rycroft, A. N., Williams, D., Cullen, J. M. \& Macdonald, J. (1991). The cytotoxin of Actinobacillus pleuropneumoniae (pleurotoxin) is distinct from the haemolysin and is associated with a $120 \mathrm{kDa}$ polypeptide. J Gen Microbiol 137, 561-568.

Sebunya, N. K. \& Saunders, J. R. (1983). Haemophilus pleuropneumoniae infection in swine: a review. Am $J$ Vet Res 182, $1331-1337$

Smits, M. A., Briaire, J., Jansen, R., Smith, H. E., Kamp, E. M. \& Gielkens, A. L. J. (1991). Cytolysins of Actinobacillus pleuropneumoniae serotype 9. Infect Immun 59, 4497-4504.

Thwaits, R. N. \& Kadis, S. (1991). Immunogenicity of Actinobacillus pleuropneumoniae outer membrane proteins and enhancement of phagocytosis by antibodies to the proteins. Infect Immun 59, 544-549.

Udeze, F. A. \& Kadis, S. (1988). Effects of Actinobacillus pleuropneumoniae hemolysin on the viability and function of porcine phagocytes. Proc Int Pig Vet Soc Congr 3, 4.

Udeze, F. A. \& Kadis, S. (1992). Effects of Actinobacillus pleuropneumoniae hemolysin on porcine neutrophil function. Infect Immun 60, 1558-1567.

Van Leengoed, L. A., Kamp, E. M. \& Pol, J. M. A. (1989). Toxicity of Haemophilus pleuropneumoniae to porcine lung macrophages. Vet Microbiol 19, 337-349.

Received 4 June 1993; accepted 2 August 1993. 